\title{
A Special Class of Mixed Constrained Optimal Control Problems
}

\author{
M. d. R. de Pinho, M. M. A. Ferreira, and F. A. C. C. Fontes
}

\begin{abstract}
The focus of this paper is on optimal control problems with mixed state and control inequality constraints. We identify a class of problems which can be associated with an auxiliary problem with both regular mixed constraints and pure state constraints. For that class of problems we derive a new set of necessary conditions of optimality.
\end{abstract}

\section{INTRODUCTION}

Consider the following optimal control problem:

$$
\left(P_{m}\right)\left\{\begin{array}{rll}
\text { Minimize } l(x(0), x(1)) & \\
\text { subject to } & & \\
\dot{x}(t) & =f(t, x(t), u(t), v(t)) & \text { a.e. } \\
0 & \geq g(t, x(t), u(t), v(t)) & \text { a.e. } \\
v(t) & \in V(t) & \text { a.e. }
\end{array}\right.
$$

with data the functions $l: \mathbb{R}^{n} \times \mathbb{R}^{n} \rightarrow \mathbb{R}, f:[0,1] \times \mathbb{R}^{n} \times$ $\mathbb{R}^{k_{u}} \times \mathbb{R}^{k_{v}} \rightarrow \mathbb{R}^{n}, g:[0,1] \times \mathbb{R}^{n} \times \mathbb{R}^{k_{u}} \times \mathbb{R}^{k_{v}} \rightarrow \mathbb{R}^{m}, \mathrm{a}$ multifunction $V:[0,1] \rightrightarrows \mathbb{R}^{k_{v}}$ and a set $C \subset \mathbb{R}^{n} \times \mathbb{R}^{n}$. We set $k=k_{u}+k_{v}$ and we concentrate on problems with $k \geq m$.

The domain of problem $\left(P_{m}\right)$ is the set of admissible processes, namely triples $(x, u, v)$ comprising measurable control functions $u$ and $v$ and a corresponding state trajectory $x \in W^{1,1}\left([0,1], \mathbb{R}^{n}\right)$ which satisfies the constraints of the problem. An admissible process is a minimizer if it minimizes the cost over admissible processes. Take a minimizer $(\bar{x}, \bar{u}, \bar{v})$ and define the set of active constraints

$$
\begin{gathered}
\mathcal{I}_{a}(t)= \\
\left\{i \in\{1, \ldots, m\}: g_{i}(t, \bar{x}(t), \bar{u}(t), \bar{v}(t))=0\right\} .
\end{gathered}
$$

The matrix:

$$
\Upsilon(t)=\nabla_{u} g^{\mathcal{I}_{a}(t)}(t, \bar{x}(t), \bar{u}(t), \bar{v}(t))
$$

where $A^{\mathcal{I}_{a}(t)}$ denotes the matrix we obtain from a matrix $A$ after removing all the rows of index $i \notin \mathcal{I}_{a}(t)$, will be of importance.

The subject of optimality necessary conditions for $\left(P_{m}\right)$ has been widely studied; see for example [1], [2], [3], [4], [5], to name but a few. Necessary conditions for $\left(P_{m}\right)$ have previously been derived under conditions directly related to the following full rank condition:

M. d. R. de Pinho and M. M. A. Ferreira are with ISR and DEEC, Faculdade de Engenharia da Universidade do Porto, Rua Dr. Roberto Frias, 4200465 Porto, Portugal. mrpinho@fe.up.pt, mmf@fe.up.pt.

F. A. C. C. Fontes is with Officina Mathematica, Departamento de Matemática para a Ciência e Tecnologia, Universidade do Minho, 4800058 Guimarães, Portugal. ffontes @mct . uminho.pt

The financial support of FEDER and FCT, Projecto POSC/EEASRI/61831/2004 is gratefully acknowledged.
FR There exists a $K>0$ such that

$$
\operatorname{det} \Upsilon(t) \Upsilon(t)^{*} \geq K \quad \text { a.e. }
$$

The full rank condition on $\Upsilon(t)$ is called a "regularity" condition and problems satisfying it are called "regular" problems.

Known necessary conditions of optimality associated with regular problems, with smooth or nonsmooth data, do not involve any measures. Derivation of optimality conditions for problems with nonregular mixed constraints remains a largely unexplored area (see [6] for references on nonregular problems).

In this paper we identify a particular class of mixed constrained optimal control problems where the mixed constraints can be split into a system of regular mixed constraints and pure state constraints. Those are problems for which the matrix $\Upsilon(t)$ may lose rank. The problem of interest is:

$$
(P)\left\{\begin{array}{rll}
\text { Minimize } l(x(0), x(1)) & \\
\text { subject to } & \\
\dot{x}(t) & =f(t, x(t), u(t)) & \text { a.e. } \\
0 & \geq D x(t)+E u(t) & \text { a.e. } \\
(x(0), x(1)) & \in C
\end{array}\right.
$$

where $D$ and $E$ are constant matrices such that $D \in \mathcal{M}_{m \times n}$, $E \in \mathcal{M}_{m \times k}$. Here $\mathcal{M}_{p \times q}$ denotes the set of all $p \times q$ matrices with real entries.

The structure of $(P)$, although simple, is convenient for our purpose since it helps simplify the exposition while highlighting the distinctive aspect of these problems. Taking into account the special structure of these problems, we derive a new set of necessary conditions for $(P)$. The noteworthy aspect of this set of necessary conditions for $(P)$ is that they can involve measures.

In this paper we work with necessary conditions in the form of Unmaximized Hamiltonian Inclusion type conditions (referred in what follows simply as UHI type conditions). UHI conditions were first derived for nonsmooth standard optimal control problems in [7] and recently they have been generalized to cover problems with both mixed constraints and pure constraints; see for example [8] and [9].

The paper is organized in the following way. In the next section we briefly present the notation used and give some definitions. Section 3 contains UHI-type conditions for general optimal control problems with both pure state constraints and mixed state control constraints. The main result in this section is a generalization of [10, Theorem 3.1] covering problems with multiple pure state constraints. It will 
be essential in our set up. In section 4 we turn to the problem of interest $(P)$. We show that $(P)$ may be associated with an "auxiliary" problem involving regular mixed constraints and pure state constraints. Furthermore, applying the result derived in section 3 we deduce new necessary conditions for $(P)$ in the form of a weak maximum principle.

\section{PRELIMINARIES}

Here and throughout, $B$ represents the closed unit ball centered at the origin and $|\cdot|$ the Euclidean norm or the induced matrix norm on $\mathbb{R}^{m \times k}$. The Euclidean distance function with respect to a set $A \subset \mathbb{R}^{k}$ is

$$
d_{A}: \mathbb{R}^{k} \rightarrow \mathbb{R}, \quad y \mapsto d_{A}(y)=\inf \{|y-x|: x \in A\} .
$$

For $r \in \mathbb{R}^{n}$, the notation $r \geq 0$ means that each component $r_{i}$ of $r$ is nonnegative. Let $A$ be a $m \times n$ real matrix with entries $a_{i j}$. Then $A \geq 0$ means $a_{i j} \geq 0$ for each $i$ and $j$.

The linear space $W^{1,1}\left([0,1] ; \mathbb{R}^{p}\right)$ denotes the space of absolutely continuous functions, $L^{1}\left([0,1] ; \mathbb{R}^{p}\right)$ the space of integrable functions and $L^{\infty}\left([0,1] ; \mathbb{R}^{p}\right)$ the space of essentially bounded functions from $[0,1]$ to $\mathbb{R}^{p}$. Also $C^{*}([0,1] ; \mathbb{R})$ is the dual of the space of continuous functions defined in $[0,1]$ and taking values in $\mathbb{R}$.

We make use of the following concepts from nonsmooth analysis. Let $A \subset \mathbb{R}^{k}$ be a closed set and $x \in A$. A vector $p \in \mathbb{R}^{k}$ is a limiting normal to $A$ at $\bar{x}$ if there exist $p_{i} \rightarrow p$ and $x_{i} \rightarrow \bar{x}$, and a sequence of positive scalars $\left\{M_{i}\right\}_{i \in \mathbb{N}}$, such that

$$
\left\langle p_{i}, x-x_{i}\right\rangle \leq M_{i}\left|x-x_{i}\right|^{2}
$$

for all $x \in A$ and for each $i \in \mathbb{N}$ (i.e., limiting normals are limits of vectors which support $A$ at points near $\bar{x}$, to second-order).

The limiting normal cone to $A$ at $\bar{x}$, written $N_{A}(\bar{x})$, is the set of all limiting normals to $A$ at $\bar{x}$.

Given a lower semicontinuous function $f: \mathbb{R}^{k} \rightarrow \mathbb{R} \cup$ $\{+\infty\}$ and a point $x \in \mathbb{R}^{k}$ such that $f(x)<+\infty$, the limiting subdifferential of $f$ at $x$, written as $\partial f(x)$, is the set

$$
\partial f(x):=\left\{\zeta:(\zeta,-1) \in N_{e p i\{f\}}(x, f(x))\right\},
$$

where $e p i\{f\}=\{(x, \eta): \eta \geq f(x)\}$ denotes the epigraph set.

In the case that the function $f$ is Lipschitz continuous near $x$, the convex hull of the limiting subdifferential, co $\partial f(x)$, coincides with the Clarke generalized subdifferential, which may be defined directly. Properties and the calculus for these various constructions may be found in [11], [12], [13].

Consider a function $h:[0,1] \times \mathbb{R}^{n} \rightarrow \mathbb{R}$. We also make use of the subdifferential $\partial_{x}^{>} h$, defined as

$$
\begin{aligned}
& \partial_{x}^{>} h(t, x)=\operatorname{co}\left\{\xi: \exists\left(t_{i}, x_{i}\right) \stackrel{h}{\rightarrow}(t, x):\right. \\
& \left.h\left(t_{i}, x_{i}\right)>0 \forall i, \nabla_{x} h\left(t_{i}, x_{i}\right) \rightarrow \xi\right\} .
\end{aligned}
$$

\section{EXTENDED UNMAXIMIZED HAMILTONIAN TYPE CONDITIONS}

We now concentrate on the more general problem

$$
\begin{aligned}
& \text { Minimize } l(x(0), x(1)) \\
& \text { subject to } \\
& \dot{x}(t)=f(t, x(t), u(t), v(t)) \quad \text { a.e. } \\
& 0 \geq g(t, x(t), u(t), v(t)) \quad \text { a.e. } \\
& 0 \geq h(t, x(t)) \quad \text { for all } t \\
& v(t) \in V(t) \\
& (x(0), x(1)) \in C
\end{aligned}
$$

where $l, f, g, V$ and $C$ are as defined before and $h:[0,1] \times$ $\mathbb{R}^{n} \rightarrow \mathbb{R}^{s}$, with $s \geq 1$.

For $(S)$ let $(\bar{x}, \bar{u}, \bar{v})$ be a reference process. Take a parameter $\varepsilon>0$. Define the set

$$
\begin{gathered}
T_{\varepsilon}(t)=\{(x, u, v): x \in \bar{x}(t)+\varepsilon B, \\
u \in(\bar{u}(t)+\varepsilon B), v \in(\bar{v}(t)+\varepsilon B) \cap V(t)\} .
\end{gathered}
$$

Definition 3.1: An admissible process $(\bar{x}, \bar{u}, \bar{v})$ for $(S)$ is a weak local minimizer if there exists a parameter $\delta>0$ such that

$$
l(x(0), x(1)) \geq l(\bar{x}(0), \bar{x}(1))
$$

over admissible processes $(x, u, v)$ satisfying

$$
(x(t), u(t), v(t)) \in T_{\delta}(t) \quad \text { a.e. }
$$

For a reference process $(\bar{x}, \bar{u}, \bar{v}), \bar{f}(t), \bar{g}(t)$, etc, denote the corresponding function evaluated at $(t, \bar{x}(t), \bar{u}(t), \bar{v}(t))$.

The following hypotheses, which make reference to a parameter $\varepsilon>0$, are imposed:

H1 The function $t \rightarrow f(t, x, u, v)$ is Lebesgue measurable for each $(x, u, v)$ and there exists a function $K_{f}$ in $L^{1}$ such that

$$
\begin{gathered}
\left|f(t, x, u, v)-f\left(t, x^{\prime}, u^{\prime}, v^{\prime}\right)\right| \\
\leq K(t)\left[\left|x-x^{\prime}\right|^{2}+\left|u-u^{\prime}\right|^{2}+\left|v-v^{\prime}\right|^{2}\right]^{1 / 2} \\
\text { for }(x, u, v),\left(x^{\prime}, u^{\prime}, v^{\prime}\right) \in T_{\varepsilon}(t) \text { a.e. } t \in[0,1] .
\end{gathered}
$$

H2 The multifunction $V$ has Borel measurable graph and

$$
V_{\varepsilon}(t):=(\bar{v}(t)+\varepsilon B) \cap V(t)
$$

is closed for almost all $t \in[0,1]$.

H3 The endpoint constraint set $C$ is closed and $l$ is locally Lipschitz in a neighbourhood of $(\bar{x}(0), \bar{x}(1))$.

H4 For $x \in \bar{x}(t)+\varepsilon B$ the function $t \rightarrow h(t, x)$ is continuous and there exists a scalar $K_{h}>0$ such that $x \rightarrow h(t, x)$ is Lipschitz of rank $K_{h}$ for all 
$t \in[0,1]$

H5 The function $t \rightarrow g(t, x, u, v)$ is Lebesgue measurable for each $(x, u, v)$.

There exists $L_{g} \in L^{1}$ such that, for almost every $t \in[0,1],(x, u, v) \rightarrow g(t, x, u, v)$ is continuously differentiable with Lipschitz constant $L_{g}(t)$ on $T_{\varepsilon}(t)$.

There exists a constant $K_{g}>0$ such that, for almost every $t \in[0,1]$,

$$
\left|\nabla_{x} \bar{g}(t)\right|+\left|\nabla_{u} \bar{g}(t)\right| \leq K_{g}
$$

H6 There exists an increasing function $\tilde{\theta}: \mathbb{R}^{+} \rightarrow \mathbb{R}^{+}$, $\tilde{\theta}(s) \downarrow 0$ as $s \downarrow 0$, such that and for all $\left(x^{\prime}, u^{\prime}, v^{\prime}\right)$, $(x, u, v) \in T_{\varepsilon}(t)$ for almost every $t \in[0,1]$,

$$
\begin{gathered}
\left|\nabla_{x, u, v} g\left(t, x^{\prime}, u^{\prime}, v^{\prime}\right)-\nabla_{x, u, v} g(t, x, u, v)\right| \\
\leq \tilde{\theta}\left(\left|\left(x^{\prime}, u^{\prime} v^{\prime}\right)-(x, u, v)\right|\right) .
\end{gathered}
$$

H7 There exists $K>0$ such that

$$
\operatorname{det} \Upsilon(t) \Upsilon^{*}(t) \geq K
$$

for almost every $t \in[0,1]$, where $\Upsilon(t)$ is as defined in (2).

Hypothesis $\mathrm{H} 7$ is condition FR of the Introduction.

For $(S)$ define the Hamiltonian to be

$$
H(t, x, p, r, u, v)=p \cdot f(t, x, u, v)+r \cdot g(t, x, u, v) .
$$

The following theorem is a generalization of Unmaximized Hamiltonian type conditions for $(S)$, proved in [10].

Theorem 3.1: Let $(\bar{x}, \bar{u}, \bar{v})$ be a weak local minimizer to problem $(S)$. Assume that $\mathrm{H} 1-\mathrm{H} 7$ are satisfied for some parameter $\varepsilon$.

Then there exists an absolutely continuous function $p$ : $[0,1] \mapsto \mathbb{R}^{n}$, integrable functions $\zeta:[0,1] \rightarrow \mathbb{R}^{k_{v}}, \gamma_{j}:$ $[0,1] \rightarrow \mathbb{R}^{n}$, for $j=1, \ldots, s$, and $r:[0,1] \rightarrow \mathbb{R}^{m}$, nonnegative Radon measures $\mu_{j} \in C^{*}([0,1], \mathbb{R}), j=1, \ldots, s$, and a scalar $\lambda \geq 0$ such that
(i) $\sum_{j=1}^{s} \mu_{j}\{[0,1]\}+\|p\|_{\infty}+\lambda>0$,
(ii) $(-\dot{p}(t), 0, \zeta(t)) \in \operatorname{co} \partial H(t, \bar{x}(t), \pi(t), r(t), \bar{u}(t), \bar{v}(t))$ a.e.
(iii) $\zeta(t) \in \operatorname{co} N_{V(t)}(\bar{v}(t))$ a.e.
(iv) $r(t) \cdot g(t, \bar{x}(t), \bar{u}(t), \bar{v}(t))=0$ and $r(t) \leq 0$ a.e.

(v) $(p(0),-\pi(1)) \in N_{C}(\bar{x}(0), \bar{x}(1))+\lambda \partial l(\bar{x}(0), \bar{x}(1))$, where

$$
\pi(t)= \begin{cases}p(t)+\int_{[0, t)} \sum_{j=1}^{s} \gamma_{i}(\tau) \mu_{j}(d \tau) \quad \text { for } t \in[0,1) \\ p(t)+\int_{[0,1]} \sum_{j=1}^{s} \gamma_{i}(\tau) \mu_{j}(d \tau) \quad \text { for } t=1, \\ \gamma_{j}(t) \in \partial_{x}^{>} h_{j}(t, \bar{x}(t)) \quad \mu_{j} \text {-a.e. }\end{cases}
$$

and

$$
\operatorname{supp}\left\{\mu_{j}\right\} \subset\left\{t \in[0,1]: h_{j}(t, \bar{x}(t))=0\right\} .
$$

Remark 3.2: Consider now $(S)$ where the pure state constraint $h(t, x) \leq 0$ is absent. Then $(S)$ is a problem with only mixed constraints and Theorem 3.1 coincides with [8, Theorem 3.1].

Proof: The Theorem can be easily established associating $(S)$ with an "auxiliary" problem where the multiple state constraints

$$
h(t, x) \leq 0
$$

is replaced by the scalar state constraint functional

$$
h^{+}(t, x)=\max \left\{h_{1}(t, x), \ldots, h_{s}(t, x)\right\} .
$$

Application of [10, Theorem 3.1] to this new problem leads to the required conclusions.

\section{UHI-TYPE CONDITIONS FOR $(P)$}

We now turn to $(P)$. Take $(\bar{x}, \bar{u})$ to be a weak local minimizer to $(P)$ (Definition 3.1 adapts easily to $(P)$ ). Consider the following additional hypotheses for $(P)$ :

AH1 The function $t \rightarrow f(t, x, u)$ is Lebesgue measurable for each pair $(x, u)$.

The function $(x, u) \rightarrow f(t, x, u)$ is continuously differentiable for almost every $t \in[0,1]$ and

$$
\left|\nabla_{x} \bar{f}(t)\right| \leq K(t), \quad\left|\nabla_{u} \bar{f}(t)\right| \leq K(t) \quad \text { a.e. }
$$

for some $K \in L^{1}$.

AH7 Matrix $E$ has rank $q$ where $1 \leq q \leq m$ and there exist nonsingular matrices $S \in \mathcal{M}_{m \times m}$ and $T \in$ $\mathcal{M}_{k \times k}$ such that

$$
S E T=\left[\begin{array}{cc}
\Sigma_{1} & \Sigma_{2} \\
0 & 0
\end{array}\right]
$$

where $\Sigma_{1} \in \mathcal{M}_{q \times q}$ is nonsingular, $\Sigma_{2} \in$ $\mathcal{M}_{q \times(k-q)}$. Furthermore

(i) $S^{-1} \geq 0$;

(ii) $S(D \bar{x}(t)+E \bar{u}(t)) \leq 0 \quad$ a.e.

Hypothesis AH1 is stronger than H1. It is introduced here to simplify the forthcoming exposition.

Remark 4.1: It is a well known fact that if $E$ is of rank $q \leq m$, then there exist nonsingular matrices $S$ and $T$ such 
that (6) holds (see [14, 0.4.6, (c)]). However, in view of AH7-i and AH7-ii, AH7 does not apply to all matrices $E$ of rank $q$.

The assumption AH7-ii depends on the optimal solution $(\bar{x}, \bar{u})$. Although AH7-ii may not be easy to check in applications it is trivially satisfied if $S$ is a diagonal matrix with nonnegative entries or when all the mixed constraints are active along the optimal solution. In the latter case it is a simple matter to see that the class of matrices $S$ satisfying AH7 contains more matrices than diagonal matrices with nonnegative entries. Take, for example,

$$
E=\left[\begin{array}{rrrr}
5 & 3 & 4 & 0 \\
3 & 2 & 0 & -4 \\
3 & 2 & 0 & -4
\end{array}\right], \quad S=\left[\begin{array}{rrr}
1 & 0 & 0 \\
-1 & 1 & 0 \\
0 & -1 & 1
\end{array}\right]
$$

The rank of $E$ is 2 and $S^{-1} \geq 0$. Then, for

$$
T=\left[\begin{array}{rrrr}
-\frac{5}{19} & -\frac{4}{19} & \frac{4}{19} & \frac{10}{19} \\
\frac{16}{19} & \frac{9}{19} & \frac{10}{19} & -\frac{13}{19} \\
-\frac{1}{19} & \frac{3}{19} & -\frac{3}{19} & \frac{2}{19} \\
-\frac{10}{19} & -\frac{8}{19} & \frac{8}{19} & \frac{1}{19}
\end{array}\right]
$$

we have

$$
S E T=\left[\begin{array}{rrrr}
1 & 1 & 2 & 1 \\
2 & 1 & -2 & -1 \\
0 & 0 & 0 & 0
\end{array}\right]
$$

Remark 4.2: If $q=m$, then matrix $E$ is of full rank and assumption $\mathrm{H} 7$ (or, what is the same, FR) is satisfied whatever $\mathcal{I}_{a}(t)$ is. In this case $(P)$ is a regular problem. On the other hand, if $q=0$, then $(P)$ can be reduced to a problem with pure state constraints. Thus the cases of interest in this paper are those when the rank $q$ of $E$ satisfies $1 \leq q<m$. It is worth mentioning that $(P)$ may be a regular problem even when $1<q<m$.

We consider the matrices $S$ and $T$ partitioned in the following way:

$$
S=\left[\begin{array}{ll}
S_{1} & S_{2} \\
S_{3} & S_{4}
\end{array}\right], \quad T=\left[\begin{array}{cc}
T_{1} & T_{2} \\
T_{3} & T_{4}
\end{array}\right],
$$

where

$$
\begin{array}{ll}
S_{1} \in \mathcal{M}_{q \times q}, & S_{2} \in \mathcal{M}_{q \times(m-q)}, \\
S_{3} \in \mathcal{M}_{(m-q) \times q}, & S_{4} \in \mathcal{M}_{(m-q) \times(m-q)}, \\
T_{1} \in \mathcal{M}_{q \times q}, & T_{2} \in \mathcal{M}_{q \times(k-q)}, \\
T_{3} \in \mathcal{M}_{(k-q) \times q}, & T_{4} \in \mathcal{M}_{(k-q) \times(k-q)} .
\end{array}
$$

Also we set

$$
\begin{array}{ll}
\hat{S}_{1}=\left[\begin{array}{ll}
S_{1} & S_{2}
\end{array}\right], & \hat{S}_{2}=\left[\begin{array}{ll}
S_{3} & S_{4}
\end{array}\right], \\
\hat{T}_{1}=\left[\begin{array}{l}
T_{1} \\
T_{3}
\end{array}\right], & \hat{T}_{2}=\left[\begin{array}{c}
T_{2} \\
T_{4}
\end{array}\right] .
\end{array}
$$

Take matrix $D$ partitioned as

$$
D=\left[\begin{array}{l}
D_{1} \\
D_{2}
\end{array}\right]
$$

where $D_{1} \in \mathcal{M}_{q \times n}$ and $D_{2} \in \mathcal{M}_{(m-q) \times n}$. Set

$$
\hat{D}_{1}=\hat{S}_{1} D, \quad \hat{D}_{2}=\hat{S}_{2} D
$$

Hypothesis AH7 shall be of the foremost importance in the analysis since it permits the reformulation of the mixed constraint

$$
D x(t)+E u(t) \leq 0
$$

into regular mixed constraints and pure state constraints as we show next.

Set $v=T^{-1} u$ with $v=\left(v_{1}, v_{2}\right) \in \mathbb{R}^{k}$ where $v_{1} \in \mathbb{R}^{q}$ and $v_{2} \in \mathbb{R}^{k-q}$. In the formulation of $(P)$ replace $u$ by $T v$ and the mixed constraint by

$$
S D x(t)+S E T v(t) \leq 0 .
$$

We obtain a new problem, denoted $\left(P_{\text {aux }}\right)$. By definition of $S$ and $T$ the inequality (7) is equivalent to

$$
\begin{cases}\hat{D}_{1} x(t)+\Sigma_{1} v_{1}(t)+\Sigma_{2} v_{2}(t) & \leq 0 \\ \hat{D}_{2} x(t) & \leq 0\end{cases}
$$

If $(\bar{x}, \bar{u})$ is a weak local minimizer to $(P)$, then, by AH7ii, we have

$$
S D \bar{x}(t)+S E \bar{u}(t) \leq 0 \quad \text { a.e. }
$$

Thus it is a simple matter to see that $\left(\bar{x}, \bar{v}_{1}, \bar{v}_{2}\right)$, where

$$
\left[\begin{array}{l}
\bar{v}_{1}(t) \\
\bar{v}_{2}(t)
\end{array}\right]=T^{-1} \bar{u}(t)
$$

is admissible for $\left(P_{a u x}\right)$.

On the other hand, if $(\tilde{x}, \tilde{v})$ is an admissible process for $\left(P_{a u x}\right)$, then AH7-i asserts that $(\tilde{x}, \tilde{u})$, with $\tilde{u}(t)=T \tilde{v}(t)$, is an admissible process for $(P)$.

We summarize our findings:

Proposition 4.3: Let $(\bar{x}, \bar{u})$ be a weak local minimizer for $(P)$. Suppose that the data of $(P)$ satisfy hypothesis AH7. Then $\left(\bar{x}, \bar{v}_{1}, \bar{v}_{2}\right)$, where

$$
\bar{v}(t)=\left[\begin{array}{c}
\bar{v}_{1}(t) \\
\bar{v}_{2}(t)
\end{array}\right]=T^{-1} \bar{u}(t)
$$

is a weak local minimizer to the problem $\left(P_{a u x}\right)$ :

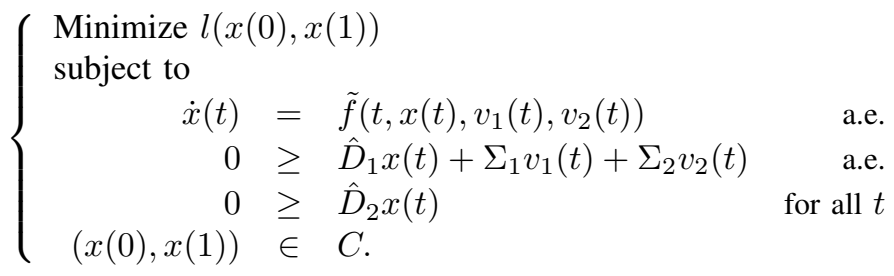

where

$$
\tilde{f}\left(t, x, v_{1}, v_{2}\right)=f\left(t, x,\left(T_{1} v_{1}+T_{2} v_{2}, T_{3} v_{1}+T_{4} v_{2}\right)\right) .
$$

Remark 4.4: For problem $\left(P_{a u x}\right)$ define the set of active mixed constraints $\mathcal{J}_{a}(t)$ as

$$
\begin{gathered}
\mathcal{J}_{a}(t)= \\
\left\{i \in\{1, \ldots, q\}: \hat{d}_{1_{i}} \bar{x}(t)+\sigma_{1_{i}} \bar{v}_{1}(t)+\sigma_{2_{i}} \bar{v}_{2}(t)=0\right\}
\end{gathered}
$$


where $\hat{d}_{1_{i}}, \sigma_{1_{i}}$ and $\sigma_{2_{i}}$ denote respectively the $i$-th row of the matrices $\hat{D}_{1}, \Sigma_{1}$ and $\Sigma_{2}$. Let $G\left(x, v_{1}, v_{2}\right)$ be the mixed constraint functional, i.e.,

$$
G\left(x, v_{1}, v_{2}\right)=\hat{D}_{1} x+\Sigma_{1} v_{1}+\Sigma_{2} v_{2} .
$$

Then

$$
\nabla_{v_{1}} G\left(x, v_{1}, v_{2}\right)=\Sigma_{1}
$$

is always of full rank independently of $\mathcal{J}_{a}(t)$, since $\Sigma_{1}$ is a nonsingular matrix. Thus $\left(P_{\text {aux }}\right)$ is a problem with regular mixed constraints

$$
\hat{D}_{1} x(t)+\Sigma_{1} v_{1}(t)+\Sigma_{2} v_{2}(t) \leq 0
$$

and pure state constraints

$$
\hat{D}_{2} x(t) \leq 0 .
$$

We are now in position to state our next result.

Theorem 4.5: Let $(\bar{x}, \bar{u})$ be a weak local minimizer to $(P)$. If the data of $(P)$ satisfy hypotheses $\mathrm{AH} 1, \mathrm{H} 3$ and AH7, then there exist an absolutely continuous function $p:[0,1] \mapsto \mathbb{R}^{n}$, integrable function $r:[0,1] \rightarrow \mathbb{R}^{m}$, nonnegative Radon measures $\mu_{j} \in C^{*}([0,1], \mathbb{R})$, with $j=$ $1, \ldots, m-q$, and a scalar $\lambda \geq 0$ such that

(i) $\sum_{j=1}^{m-q} \mu_{j}\{[0,1]\}+\|p\|_{\infty}+\lambda>0$,

(ii) $\quad-\dot{p}(t)=\pi(t) \cdot \nabla_{x} f(t, \bar{x}(t), \bar{u}(t))+r(t) \cdot D$ a.e.

(iii) $0=\pi(t) \cdot \nabla_{u} f(t, \bar{x}, \bar{u})+r(t) \cdot E$ a.e.

(iv) $r(t) \cdot(D \bar{x}(t)+E \bar{u}(t))=0$ a.e.

(v) $(p(0),-\pi(1)) \in N_{C}(\bar{x}(0), \bar{x}(1))+\lambda \partial l(\bar{x}(0), \bar{x}(1))$,

where

$$
\operatorname{supp}\left\{\mu_{j}\right\} \subset\left\{t \in[0,1]: \hat{d}_{2_{j}} \bar{x}(t)=0\right\},
$$

and $\pi$ is defined as

$$
\pi(t)= \begin{cases}p(t)+\int_{[0, t)} \sum_{j=1}^{m-q} \hat{d}_{2_{j}} \mu_{j}(d \tau) & \text { for } t \in[0,1) \\ p(t)+\int_{[0,1]} \sum_{j=1}^{m-q} \hat{d}_{2_{j}} \mu_{j}(d \tau) & \text { for } t=1 .\end{cases}
$$

with $\hat{d}_{2_{j}}$ being the j-th row of $\hat{D}_{2}$.

An analogous of Theorem 4.5 can be easily obtained when $\mathrm{AH} 1$ is replaced by $\mathrm{H} 1$. As pointed out before, we choose to impose AH1 instead of $\mathrm{H} 1$ to simplify the analysis.

Notice that the multiplier $r$ in the Theorem above is not nonpositive as one may expected in view of Theorem 3.1. However, and as it will be clear in the proof, $r$ is defined as

$$
r(t)=S^{*} \hat{r}(t)
$$

with $\hat{r}(t) \leq 0$ for almost every $t \in[0,1]$.

Proof: Proposition 4.3 asserts that $\left(\bar{x}, \bar{v}_{1}, \bar{v}_{2}\right)$, where

$$
\bar{v}(t)=\left[\begin{array}{l}
\bar{v}_{1}(t) \\
\bar{v}_{2}(t)
\end{array}\right]=T^{-1} \bar{u}(t),
$$

is a weak local minimizer to $\left(P_{\text {aux }}\right)$.

It is a simple matter to see that the data of $\left(P_{\text {aux }}\right)$ satisfy the conditions under which Theorem 3.1 applies. In particular, H7 is satisfied independently of the set of active constraints.

Define the Hamiltonian for $\left(P_{\text {aux }}\right)$ to be

$$
\begin{gathered}
\tilde{H}\left(t, x, p, \tilde{r}, v_{1}, v_{2}\right)= \\
p \cdot \tilde{f}\left(t, x, v_{1}, v_{2}\right)+\tilde{r} \cdot\left(\hat{D}_{1} x+\Sigma_{1} v_{1}+\Sigma_{2} v_{2}\right) .
\end{gathered}
$$

Application of Theorem 3.1 to $\left(P_{\text {aux }}\right)$ asserts the existence of an absolutely continuous function $p:[0,1] \mapsto \mathbb{R}^{n}$, integrable function $\tilde{r}:[0,1] \rightarrow \mathbb{R}^{q}$, nonnegative Radon measures $\mu_{j} \in C^{*}([0,1], \mathbb{R}), j=1, \ldots, m-q$, and a scalar $\lambda \geq 0$ such that
(a) $\sum_{j=1}^{m-q} \mu_{j}\{[0,1]\}+\|p\|_{\infty}+\lambda>0$,
(b) $(-\dot{p}(t), 0,0)=$
$\nabla_{x, v_{1}, v_{2}} H\left(t, \bar{x}(t), \pi(t), \tilde{r}(t), \bar{v}_{1}(t), \bar{v}_{2}(t)\right)$ a.e.
(c) $\tilde{r}(t) \cdot\left(\hat{D}_{1} \bar{x}(t)+\Sigma_{1} \bar{v}_{1}(t)+\Sigma_{2} \bar{v}_{2}(t)\right)=0$ and $\tilde{r}(t) \leq 0$ a.e.

(d) $(p(0),-\pi(1)) \in N_{C}(\bar{x}(0), \bar{x}(1))+\lambda \partial l(\bar{x}(0), \bar{x}(1))$,

where (10) is satisfied and $\pi$ is defined as in (11).

Setting

$$
\begin{aligned}
A(t) & =\nabla_{x} f(t, \bar{x}(t), \bar{u}(t)), \\
B_{1}(t) & =\nabla_{u} f(t, \bar{x}(t), \bar{u}(t)) \hat{T}_{1}, \\
B_{2}(t) & =\nabla_{u} f(t, \bar{x}(t), \bar{u}(t)) \hat{T}_{2},
\end{aligned}
$$

we focus on (b). We have

$$
\begin{aligned}
-\dot{p}(t) & =A^{*}(t) \pi(t)+\hat{D}_{1}^{*} \tilde{r}(t) \\
0 & =B_{1}^{*}(t) \pi(t)+\Sigma_{1}^{*} \tilde{r}(t) \\
0 & =B_{2}^{*}(t) \pi(t)+\Sigma_{2}^{*} \tilde{r}(t)
\end{aligned}
$$

Define

$$
\hat{r}(t)=\left[\begin{array}{c}
\tilde{r}(t) \\
0
\end{array}\right] .
$$

Then (12) can be written as

$$
-\dot{p}(t)=A^{*}(t) \pi(t)+D^{*} S^{*} \hat{r}(t) .
$$

and (13) and (14) as

$$
T^{*} \nabla_{u} f(t, \bar{x}(t), \bar{u}(t))^{*} \pi(t)+T^{*} E^{*} S^{*} \hat{r}(t)=0 .
$$


Since $T$ is nonsingular we deduce from (17) that

$$
0=\nabla_{u} f(t, \bar{x}(t), \bar{u}(t))^{*} \pi(t)+E^{*} S^{*} \hat{r}(t) .
$$

Let $r(t)=S^{*} \hat{r}(t)$. From the above, we deduce that (16) and (18) are respectively

$$
\begin{aligned}
-\dot{p}(t) & =\nabla_{x} f(t, \bar{x}(t), \bar{u}(t))^{*} \pi(t)+D^{*} r(t), \\
0 & =\nabla_{u} f(t, \bar{x}(t), \bar{u}(t))^{*} \pi(t)+E^{*} r(t) .
\end{aligned}
$$

Conclusions (ii) and (iii) of the Theorem are respectively (19) and (20).

Also (a) and (d) above are respectively (i) and (v) of the Theorem.

It remains to prove (iv) of the Theorem. Taking into account the definition of $r$ and AH7-i, (c) can be rewritten as

$$
r(t) \cdot(D \bar{x}(t)+E \bar{u}(t))=0 .
$$

This is (iv) of the Theorem.

\section{REFERENCES}

[1] M. R. Hestenes, Calculus of Variations and Optimal Control. Wiley, New York, 1966.

[2] A. A. Milyutin and N. P. Osmolovskii, Calculus of Variations and Optimal Control Theory. Providence, Rhode Island: American Mathematical Society, 1998.

[3] L. W. Neustadt, Optimization, A Theory of Necessary Conditions. New Jersey: Princeton University Press, 1976.

[4] Z. Pales and V. Zeidan, "First and second order necessary conditions for control problems with constraints," Transc. American Mathematical Society, vol. 346, pp. 421-453, 1994.

[5] G. Steffani and P. Zezza, "Optimality conditions for constrained control problems," SIAM Journal of Control and Optimization, vol. 34, pp. 635-659, 1996.

[6] A. V. Dmitruk, "Maximum principle for the general optimal control problem with phase and regular mixed constraints," Computation Mathematics and Moddeling, CMMOEA, vol. 4, pp. 307-426, 1993.

[7] M. de Pinho and R. Vinter, "An Euler-Lagrange inclusion for optimal control problems," IEEE Trans. Aut. Control, vol. 40, pp. 1191-1198, 1995.

[8] M. de Pinho, "Mixed constrained control problems," Journal of Mathematical Analysis and Applications, vol. 278, pp. 293-307, 2003.

[9] M. de Pinho, M. M. A. Ferreira, and F. A. C. C. Fontes, "Unmaximized inclusion necessary conditions for nonconvex constrained optimal control problems," ESAIM, COCV Control, Optimisation and Calculus of Variations, vol. 11, pp. 614-632, 2005.

[10] —, "Unmaximized necessary conditions for constrained control problems," in Proceeding of the 43rd IEEE Conference on Decision and Control, Paradise Island, Bahamas, 14-17 Dec. 2004, 2004, pp. 917- 922.

[11] F. H. Clarke, Optimization and Nonsmooth Analysis. Wiley, New York, 1983.

[12] R. T. Rockafellar and B. Wets, Variational Analysis. Springer, Berlin, 1998.

[13] R. B. Vinter, Optimal Control. Birkhouser, 2000.

[14] R. A. Horn and C. R. Jonhson, Matrix Analysis. Cambridge Press, 1985. 